\title{
Biomicroscopia ultra-sônica (UBM) no planejamento cirúrgico e acompanfiamento melanomas malignos de íris e corpo ciliar pós-operatório de
}

Ultrasound biomicroscopy (UBM) in the surgical planning and postoperative follow-up
of iris and ciliary body melanomas

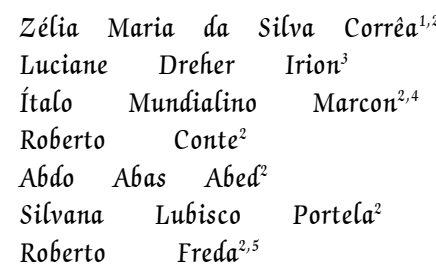

Este trabalho foi realizado no Serviço de Oftalmologia da Santa Casa de Porto Alegre e Hospital Banco de Olhos

' Responsável pelos Departamentos de Oncologia e Ultra-som do Serviço de Oftalmologia da Irmandade da Santa Casa de Misericórdia de Porto Alegre (ISCMPA) Fundação Faculdade Federal de Ciências Médicas de Porto Alegre (FFFCMPA) - RS. Pós-graduanda, nível doutorado da Universidade Federal de São Paulo (UNIFESP-EPM) - SP.

2 Oftalmologista do Corpo Clínico do Hospital Banco de Olhos, Porto Alegre- RS.

${ }_{3}^{3}$ Mestra em Anatomia Patológica pela Fundação Faculdade Federal de Ciências Médicas de Porto Alegre e Patologista do Laboratório Patologistas Reunidos, Porto Alegre - RS, Brasil.

${ }^{4}$ Chefe do Serviço de Oftalmologia da Irmandade da Santa Casa de Misericórdia de Porto Alegre / Fundação Faculdade Federal de Ciências Médicas de Porto Alegre e Livre Docente do Departamento de Oftalmo-Otorrinolaringologia da Fundação Faculdade Federal de Ciências Médicas de Porto Alegre - Porto Alegre - RS.

${ }_{5}^{5}$ Mestre em Oftalmologia pela Universidade Federal de São Paulo (UNIFESP-EPM) - SP.

Endereco para correspondência: Av. Nilo Peçanha, 2421 - Porto Alegre (RS) - CEP 91330-001

E-mail:zmcorrea@terra.com.br

Recebido para publicação em 18.09.2001

Aceito para publicação em 27.11.2002

Nota Editorial: Pela análise deste trabalho e por sua anuência na divulgação desta nota, agradecemos às Dras. Norma Allemann e Maria Carmen Menezes Santos Torres

\section{RESUMO}

Objetivo: Estudar os achados da biomicroscopia ultra-sônica (UBM) em melanomas malignos primários da íris e corpo ciliar antes e depois da ressecção cirúrgica e correlacionar a extensão do tumor (revelado pela biomicroscopia ultra-sônica) aos achados histopatológicos. Métodos: Estudo prospectivo de 5 pacientes com diagnóstico clínico de tumor de íris e/ou corpo ciliar. Estes pacientes foram avaliados pela biomicroscopia ultrasônica antes e após iridociclectomia ou iridectomia. Os espécimens foram enviados para estudo histopatológico. Os pacientes foram acompanhados por período mínimo de 6 meses quando foi realizada nova biomicroscopia ultra-sônica. Resultados: Neste grupo de 5 pacientes, 3 eram do sexo feminino e 2, do masculino, variação de idade de 32 e 64 anos. Todos os casos eram unilaterais. Quatro pacientes apresentaram massa iridociliar e um apresentou lesão limitada à íris. Em todos os casos a biomicroscopia ultra-sônica auxiliou o planejamento cirúrgico para a ressecção tumoral com margens livres. A biomicroscopia ultra-sônica no pós-operatório revelou ausência de crescimento tumoral em todos os casos, sendo que 2 pacientes apresentaram vítreo na incisão cirúrgica, 1 deles com tração vítrea leve, mas sem sinais de descolamento de retina. Conclusão: A biomicroscopia ultra-sônica pareceu ser método auxiliar confiável para diagnosticar tumores de íris e corpo ciliar, planejar o tratamento cirúrgico e o acompanhamento pós-operatório.

Descritores: Íris/ultra-sonografia; Corpo ciliar/ultra-sonografia; Neoplasias da íris/ultrasonografia; Neoplasias da íris/cirurgia; Neoplasias uveais/ultra-sonografia; Melanoma; Epitélio pigmentar ocular/ultra-sonografia

INTRODUÇÃO

O melanoma maligno da úvea é a neoplasia primária intra-ocular mais freqüente em adultos. Dos melanomas da úvea, $15 \%$ são tumores de íris e corpo ciliar $^{(1)}$. Estas lesões podem ser confundidas clinicamente com cistos, corpos estranhos retidos ou mesmo sinéquias ${ }^{(2-4)}$. O diagnóstico preciso e conhecimento do tamanho da lesão são fatores decisivos na escolha terapêutica $^{(2,5)}$. Comparados aos melanomas da úvea posterior, os melanomas de íris costumam ser pequenos e visíveis, por isto são diagnosticados precocemente ${ }^{(5)}$. Os melanomas de corpo ciliar, por outro lado, são dados como mais malignos e apresentam metástases com maior freqüência. Sua posição anatômica permite que se esconda atrás da íris por muito tempo até que esteja relativamente grande ${ }^{(6)}$. 
410 Biomicroscopia ultra-sônica (UBM) no planejamento cirúrgico e acompanhamento pós-operatório de melanomas malignos de íris e corpo ciliar

Os melanomas malignos da úvea anterior podem ser tratados de várias maneiras como: observação (em casos de tumores pequenos e de diagnóstico clínico/ultra-sonográfico incerto), ressecção cirúrgica do tumor (iridectomia ou iridociclectomia), braquiterapia com placas epibulbares e mesmo enucleação nos casos de tumores muito grandes ou com comprometimento escleral ou extra-escleral ${ }^{(5)}$. Além da extirpação do tumor, a ressecção tem a vantagem de fornecer comprovação diagnóstica com a histopatologia e evitar os efeitos secundários da radioterapia ${ }^{(6-7)}$.

A biomicroscopia ultra-sônica (UBM) tem sido um grande avanço no diagnóstico das patologias do segmento anterior, especialmente quando há envolvimento do corpo ciliar ${ }^{(8)}$. Esta tecnologia desenvolvida por Pavlin no início da década de $90^{(9)}$, está disponível comercialmente há cerca de 7 anos. $\mathrm{O}$ UBM utiliza-se de um transdutor de $50 \mathrm{MHz}$, permitindo examinar as estruturas oculares com profundidade até $5 \mathrm{~mm}$ e resolução máxima de $50 \mu \mathrm{m}$, considerada microscópica ${ }^{(6,8-9)}$. O UBM é um método de muito valor na avaliação dos tumores de segmento anterior. Este método oferece informação precisa sobre a localização e dimensões das lesões que são muito pequenas para serem avaliadas por outras técnicas ${ }^{(9-10)}$. Apesar de várias publicações já terem demonstrado a utilidade do UBM no diagnóstico de tumores do segmento anterior ${ }^{(1,6,8-11)}$, nenhum deles usou esta tecnologia no acompanhamento pósoperatório e monitorização da recorrência tumoral.

O objetivo deste artigo é estudar os achados da biomicroscopia ultra-sônica (UBM) em melanomas malignos primários da íris e corpo ciliar antes e após ressecção cirúrgica e correlacionar a extensão do tumor ao tipo celular encontrado no estudo histopatológico.

\section{MÉTODOS}

Estudo prospectivo de 5 pacientes com diagnóstico clínico e citológico ( 2 casos) de melanoma de íris e/ou corpo ciliar. Estes pacientes foram avaliados pela biomicroscopia ultrasônica (UBM) antes e após iridociclectomia e iridectomia. (vide tabela)

O UBM foi realizado conforme técnica previamente descri$\mathrm{ta}^{(8,10)}$. O aparelho usado, disponível comercialmente, foi o Biomicroscópio Ultra-sônico modelo $\mathrm{P} 40^{\circledR}$, Paradigm Industries, Salt Lake City, UT. Este aparelho tem uma sonda de 50 $\mathrm{MHz}$ com resolução máxima de $50 \mu \mathrm{m}$ e penetração de até $5 \mathrm{~mm}$. A varredura do UBM obtém imagens com dimensões de 5 x 5 mm e 256 linhas de resolução numa velocidade de 8 quadros por segundo ${ }^{(6)}$. Os exames foram realizados pelo mesmo médico (ZMSC) com o paciente posicionado em decúbito dorsal horizontal, sob anestesia tópica com cloridrato de proximetacaína (Anestalcon ${ }^{\circledR}$ ou Visonest ${ }^{\circledR}$ ). Foi colocado um espéculo palpebral acrílico em forma de anel, com $20 \mathrm{~mm}$ de diâmetro para manter as pálpebras abertas e conter o material viscoelástico (metilcelulose 2\%) usado para imersão do transdutor do ultra-som durante o exame. O transdutor, preso a um braço articulado, capturava as imagens durante o exame por meio de movimentos oscilatórios enquanto estava imersa no material viscoelástico. Os cortes realizados foram: axiais (perpendicular à córnea em sua área central)-horizontal e vertical, radiais e transversais ao limbo no sentido horário com ênfase especial na região do tumor. O tumor em questão foi medido repetidas vezes para serem obtidas suas dimensões: antero-posterior (ou radial, perpendicular ao limbo esclero-corneano), circunferencial (ou tranversal, paralelo ao limbo esclero-corneano) e espessura. Além das dimensões do tumor, foram observadas: a arquitetura das estruturas da úvea anterior bem como a diferença entre a ecogenicidade do tecido tumoral e o normal, e a possibilidade de invasão da esclera (o tecido tumoral apareceria hipoecogênico em relação à esclera). Após o exame, o espéculo foi removido e o olho examinado, lavado com soro fisiológico. As imagens foram então impressas para análise e planejamento cirúrgico.

Dois pacientes (casos 1 e 3 ) optaram por realizar biópsia aspirativa com agulha fina antes da ressecção cirúrgica do tumor. A técnica usada neste procedimento foi descrita em artigo publicado ${ }^{(12)}$ e não tem relevância neste estudo uma vez que não foi observada semeadura tumoral intra-ocular ou subconjuntival até o término do estudo. Em ambos os casos, a citologia foi positiva para células tumorais (melanoma maligno primário da úvea).

A ressecção cirúrgica foi realizada sob anestesia peribulbar com sedação, sempre pelo mesmo cirurgião (ZMSC). A iridectomia realizada foi setorial com remoção do quadrante da íris onde estava localizada a lesão através de uma incisão esclero-corneana auto-selante com $12 \mathrm{~mm}$ de comprimento. Este procedimento foi indicado quando o tumor estava restrito à íris e não comprometia o ângulo da câmara anterior ou os processos ciliares ${ }^{(13)}$. As iridociclectomias foram conforme a técnica publicada por Peyman et $\mathrm{al}^{(14)}$ com algumas particularidades descritas a seguir. Após uma peritomia conjuntival $180^{\circ}$ na região onde se localizava o tumor, foi realizada uma incisão perpendicular à esclera com bisturi de diamante usado em ceratotomia radial. Esta incisão, com profundidade estimada permitiu a confecção de um retalho escleral de aproximadamente $2 / 3$ de espessura usando uma lâmina de bisturi $n^{0} 15$. $O$ retalho de esclera foi feito com um "degrau" interno para posterior fechamento da incisão como um alçapão. Em seguida, foi realizada diatermia no leito do retalho e colocação de 2 pontos prévios com seda 4-0. A ressecção em bloco da lesão tumoral posterior, com propósito de obter cerca de $4 \mathrm{~mm}$ de margem livre e a esclera interna adjacente, foi feita com tesoura de córnea a partir de uma esclerotomia. A porção do tumor na íris foi ressecado a partir de uma iridectomia setorial com $2 \mathrm{~mm}$ a mais nas bordas do tumor também com objetivo de obter margens cirúrgicas livres no histopatológico. O tamanho exato e o local da ressecção foram calculados com base no UBM pré-operatório. Após a remoção do tumor, a ferida cirúrgica foi suturada com fio mononylon 9-0. O segmento posterior foi reconstituído com bolha de ar e vitrectomia anterior (nos 4 casos de irido- 


\begin{tabular}{|c|c|c|c|c|c|c|c|c|c|c|}
\hline Caso & $\operatorname{sex} 0$ & idade & olho & local-tu & AV pré & local-anatômico & tipo cel & AV pós & vítreo & segui/o \\
\hline 1 & Masc & 32 & D & T S & $20 / 200$ & ír-cc & misto & $20 / 200$ & não & $20 \mathrm{~m}$ \\
\hline 2 & Fem & 45 & D & $\mathrm{NI}$ & $20 / 20$ & ír-cc & misto & $20 / 20$ & não & $14 \mathrm{~m}$ \\
\hline 3 & Fem & 47 & $E$ & TI & $20 / 40$ & ír-cc & epitelióide & $20 / 60$ & $\operatorname{sim}$ & $22 \mathrm{~m}$ \\
\hline 4 & Fem & 52 & $\mathrm{E}$ & TI & $20 / 25$ & ír-cc & misto & $20 / 60$ & não & $16 \mathrm{~m}$ \\
\hline 5 & Masc & 64 & $\mathrm{E}$ & TI & $20 / 40$ & íris & fusiforme $B$ & $20 / 40$ & não & $10 \mathrm{~m}$ \\
\hline
\end{tabular}

ciclectomia), feita com tesoura Vannas e esponja de vítreo. A sutura conjuntival em pontos separados foi feita com fio poliglactina-910 $\left(\right.$ Vycryl $\left.^{\circledR}\right)$ 7-0.

Todos os espécimens foram conservados em solução com formol a $20 \%$ e enviados para estudo histopatológico. Foi prescrita a associação de antibiótico e esteróide tópico pósoperatório por 30 dias. Os pacientes foram acompanhados por um período mínimo de 6 meses quando foi realizado novo UBM com a mesma técnica anteriormente descrita, mas buscando avaliar a cicatriz cirúrgica e uma possível recorrência do tumor. Todos os pacientes permanecem em acompanhamento oftalmológico trimestral e oncológico semestral para pesquisa de metástases sistêmicas.

Os dados pessoais, clínicos, resultado do exame histopatológico e achados ao UBM pré e pós-operatório foram computados. (vide tabela)

\section{RESULTADOS}

Neste grupo de 5 pacientes, 3 eram do sexo feminino e 2 eram do masculino, com idades que variaram entre 32 e 64 anos. Todos os pacientes apresentaram tumores unilaterais. Quatro pacientes apresentaram massa iridociliar e apenas um apresentou lesão limitada à íris (Fig.1). O UBM revelou lesões de superfície irregular exceto naquela limitada à íris. Observou-se que os tumores apresentavam uma hiperecogenicidade de padrão linear na superfície com gradual atenuação sonora causada pelo tecido tumoral em todos os casos. Os tumores estudados eram sólidos com exceção do paciente $n^{0} 1$ que continha 2 cavidades císticas em seu interior. As dimensões tumorais foram medidas em todos os casos exceto na paciente $\mathrm{n}^{0} 3$ (Fig. 2) cuja espessura tumoral (aproximadamente 5,5 mm) não foi medida com precisão pelo UBM. As lesões iridociliares variaram entre 3,4 e 4,7 mm de diâmetro e espessuras entre 3,1 e 5,5 mm e a lesão de íris mediu 2,9 x 3,7 mm de base e $1,9 \mathrm{~mm}$ espessura ao UBM.

Foram realizadas 4 iridociclectomias e 1 iridotomia (caso $\mathrm{n}^{0} 5$ ). Em todos os casos, o planejamento cirúrgico para a ressecção tumoral foi baseado nas informações obtidas no UBM quanto à dimensão do tumor e à presença de comprometimento, ou não, do corpo ciliar. O retalho escleral com espes- sura de $2 / 3$ da esclera foi individualizado baseado na espessura medida ao UBM.

Durante o ato operatório, dois pacientes apresentaram hemorragia vítrea leve que se resolveu sem necessidade de intervenção ( $\operatorname{casos} n^{0} 3$ e 4).

O exame histopatológico, corado com hematoxilina-eosina, revelou 1 melanoma de íris do tipo fusiforme B (Fig. 1), 1 melanoma iridociliar do tipo epitelióide (Fig. 2) e 3 melanomas iridociliares do tipo misto (Fig. 3). Todos os espécimens $(n=5)$ apresentaram margens cirúrgicas livres e as medidas da lesão fixada em formol foi cerca de 8 a $10 \%$ menores que o estimado pelo UBM. O único tumor restrito à íris (caso $n^{0} 5$ ) era do tipo fusiforme B (Fig. 1) e o tumor com maior extensão no corpo ciliar (caso $\mathrm{n}^{0} 3$ ) era do tipo epitelióide (Fig. 2).

O UBM no $6^{\circ}$ mês pós-operatório revelou ausência de crescimento tumoral em todos os casos. Em 2 casos foi observado vítreo na incisão cirúrgica percebido ao UBM como traves lineares de média ecogenicidade que se direcionam ao local da ferida operatória. Entretanto somente em 1 caso pareceu apresentar tração e encarceramento do vítreo neste local.

A acuidade visual deste grupo de pacientes estudados antes e depois do tratamento cirúrgico está exposta na tabela anexa. A acuidade visual de 4 dos 5 pacientes apresentou-se inalterada no $6^{\circ}$ mês pós-operatório se comparada à pré-operatória. Neste período, uma paciente (caso $n^{0} 3$ ) apresentou baixa visual no pós-operatório (2 linhas na tabela de Snellen) devido à opacidade periférica do cristalino possivelmente por compressão tumoral (Fig. 2). O seguimento prolongado destes pacientes revelou mais um caso de perda visual devido a edema cístico da mácula de início tardio, aos 14 meses de pósoperatório (caso $n^{0} 4$ ), atualmente em tratamento clínico com acuidade visual corrigida de 20/60 (vide tabela). Coincidentemente, nestes 2 casos com perda visual pós-operatória, foi detectada a presença de vítreo na incisão cirúrgica, sendo que, não havia tração vítrea periférica no caso que evoluiu com edema cístico da mácula $\left(\operatorname{casos} n^{0} 4\right)$, mas esta paciente foi a única da série que se queixou de fotofobia quase intolerável durante o período pós-operatório recente.

Os pacientes estão em acompanhamento, o seguimento médio neste grupo é atualmente de 16,4 meses. Não houve complicação do tipo descolamento de retina/coróide ou recorrência tumoral até o presente momento. 


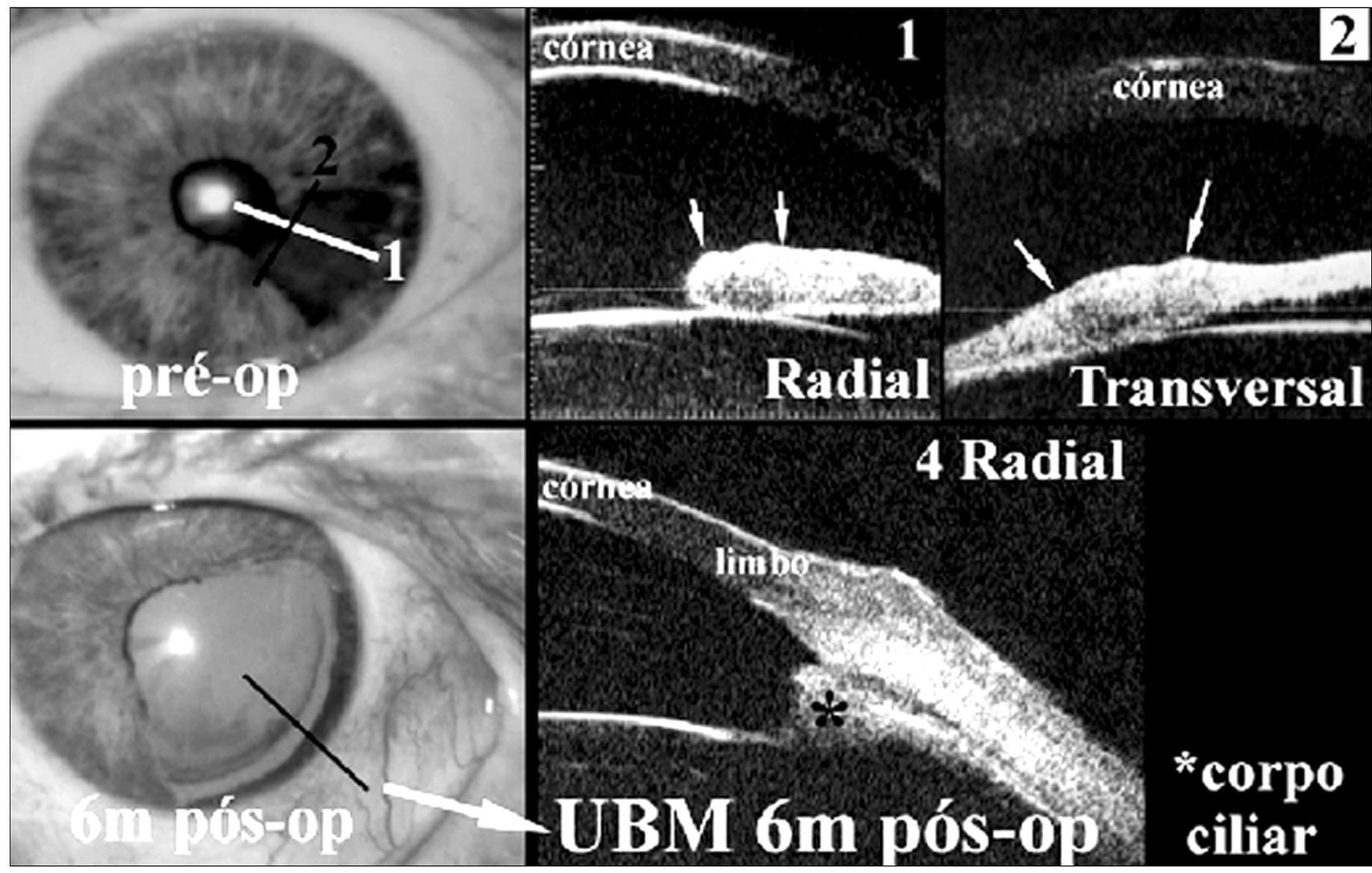

Figura 1 - Imagens digitalizadas do aspecto pré e após iridectomia do caso $n^{0} 5$. A fotografia do segmento anterior (esquerda/superior) demonstra uma lesão melanocítica, restrita a íris, única neste grupo de pacientes, com discoria e sem comprometer o ângulo da câmara anterior. Observe ao UBM (corte $1=$ radial, corte 2 = transversal) a hiperecogenicidade linear da superfície do tumor bem como sua superfície irregular apontada por flechas (centro e direita/superior). 0 aspecto no $6^{\circ}$ mês pós-operatório demonstra ampla iridectomia e ausência de recorrência do tumor (esquerda/inferior). Observe no UBM (direita/inferior) que, neste caso, o corpo ciliar foi poupado.

DISCUSSÃO

Os estudos publicados a respeito da UBM em tumores da úvea anterior demonstraram que esta modalidade ultrasonográfica pode delimitar as margens tumorais e extensão para estruturas vizinhas no segmento anterior e esclera ${ }^{(1,6,8-9)}$. Aqui também foi possível comprovar a utilidade e importância da UBM no planejamento cirúrgico destas lesões especialmente as que comprometem o corpo ciliar (Fig.1,2,3). Além disto, esta tecnologia permite também diferenciar lesões sólidas e císticas ${ }^{(2-4)}$ ou sólidas com cavidades císticas em seu interior (Fig.3).

Marigo e colaboradores descreveram as características destes tumores ao UBM e correlacionaram-nas com os achados patológicos após ressecção ou enucleação ${ }^{(6)}$. Seus resultados são semelhantes àqueles encontrados nesse estudo. Em casos de massas intra-oculares do segmento anterior é importante estudar cada meridiano do olho para poder dimensionar a extensão tumoral com segurança ${ }^{(6)}$. A apresentação e a extensão dos tumores avaliados pelo UBM neste estudo, coincidiu com o potencial invasivo revelado pela histopatologia uma vez que o único tumor restrito à íris era do tipo fusiforme B (Fig. 1) e o tumor com maior extensão no corpo ciliar era epitelióide (Fig. 2). Deve-se lembrar que os melanomas do tipo fusiforme A são considerados por muitos autores como nevos ${ }^{(15)}$ mas existem os que acreditam num potencial mínimo de malignidade uma vez que estas lesões costumam crescer durante longos períodos de acompanhamento mas não costumam originar metástases à distância ${ }^{(16)}$. Os melanomas fusiforme B são considerados melanomas de baixa malignidade, e os mistos que apresentam células fusiformes B e epitelióides. Os melanomas do tipo epitelióide são mais agressivos que os anteriores e com maior potencial metastático. Freqüentemente são observadas figuras mitóticas que sugerem a rápida multiplicação celular neste tipo de tumor $^{(16)}$. Portanto, parece lógico que o maior tumor observado neste grupo de pacientes tenha sido diagnosticado como epitelióide no exame histopatológico. O fato das lesões terem apresentado medidas um pouco menores quando fixadas pode ser explicado pela desidratação do tecido embebido no formol e também a precisão milimétrica do UBM quando comparado à medida feita pela régua do patologista. Além disto, o fato de todos os pacientes terem apresentado margens cirúrgicas livres 

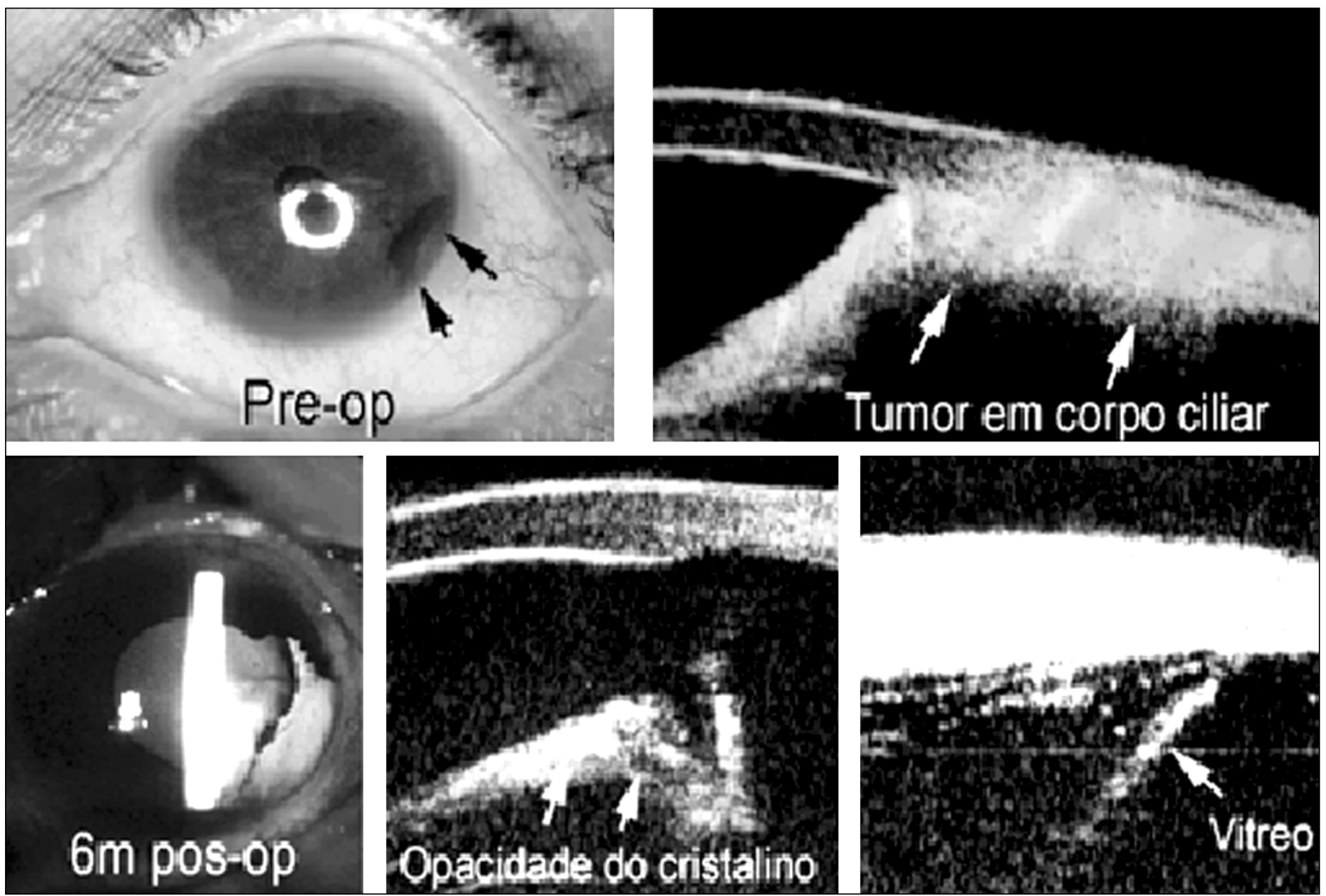

Figura 2 - Imagens digitalizadas do caso $\mathrm{n}^{0} 3$ onde se observa (esquerda/superior) uma fotografia do segmento anterior $0 \mathrm{E}$ demonstrado a lesão melanocítica temporal inferior. No UBM nota-se o comprometimento do corpo ciliar e do ângulo da câmara anterior. 0 aspecto 6 meses após iridociclectomia (esquerda/inferior) mostra a presença de uma catarata periférica provavelmente devido à compressão do cristalino pela massa tumoral. Ao UBM observa-se a hiperecogenicidade periférica do cristalino bem como a ausência de recorrência do tumor (centro/inferior). Neste caso, há vítreo na incisão cirúrgica exercendo leve tração neste local (direita/inferior)

confirma a eficiência do UBM para estimar as dimensões de tumores iridociliares.

Uma outra possibilidade terapêutica para os casos de melanomas com comprometimento da raiz da íris e corpo ciliar (casos 1 a 4) seria a braquiterapia com placa epibulbar ${ }^{(5)}$. A braquiterapia tem a vantagem de evitar uma cirurgia intraocular com suas complicações e morbidade ocular reconhecidas ${ }^{(7)}$. Entretanto, os efeitos colaterais da irradiação aparecem mais tarde, geralmente a partir de 1 ano do tratamento, e podem ser desde uma catarata até a possibilidade de sinéquias anteriores e glaucoma neovascular ${ }^{(7)}$. A ressecção tumoral em bloco tem a vantagem da comprovação histológica do diagnóstico e o conhecimento das margens cirúrgicas ressecadas (livres ou não) ${ }^{(6)}$, entretanto, a cirurgia intra-ocular também oferece riscos de complicações intra e pós-operatórias: sinéquias anteriores, tração vítreo-retininana, hemorragia vítrea e hifema, catarata por trauma cirúrgico e fotofobia devido a iridectomia ${ }^{(13)}$. Nesta série de casos, as complicações observadas até o presente foram mínimas, restritas a hemorragias vítreas leves que se resolveram sem reintervenção (casos n ${ }^{0} 3$ e 4), 1 caso de tração vítreo-retiniana aparentemente sem transtornos retinianos (caso $\mathrm{n}^{0} 3$ ) e 1 caso de edema cístico de mácula na paciente que referiu fotofobia pós-operatória mais intensa no grupo (caso $n^{0} 4$ ). Moreira e colaboradores descreveram um caso de melanoma maligno de coróide periférico, com margem posterior no equador do olho, submetido à ressecção cirúrgica em bloco que evoluiu com edema cístico de mácula pós-operatório ${ }^{(17)}$.

Foi interessante observar as características intra-oculares após as ressecções iridociliares utilizando o UBM. Achados como o aspecto das margens cirúrgicas, do retalho escleral e a presença de vítreo na incisão cirúrgica, confirmam a importância deste método diagnóstico auxiliar no acompanhamento após procedimentos desse porte. Indícios clínicos de recorrência tumoral como pigmentação da esclera, que pode ser detectada ao exame clínico, elevações no corpo ciliar e/ou irregularidade na margem interna da ferida cirúrgica detectadas ao UBM, não foram detectados até o presente momento. 

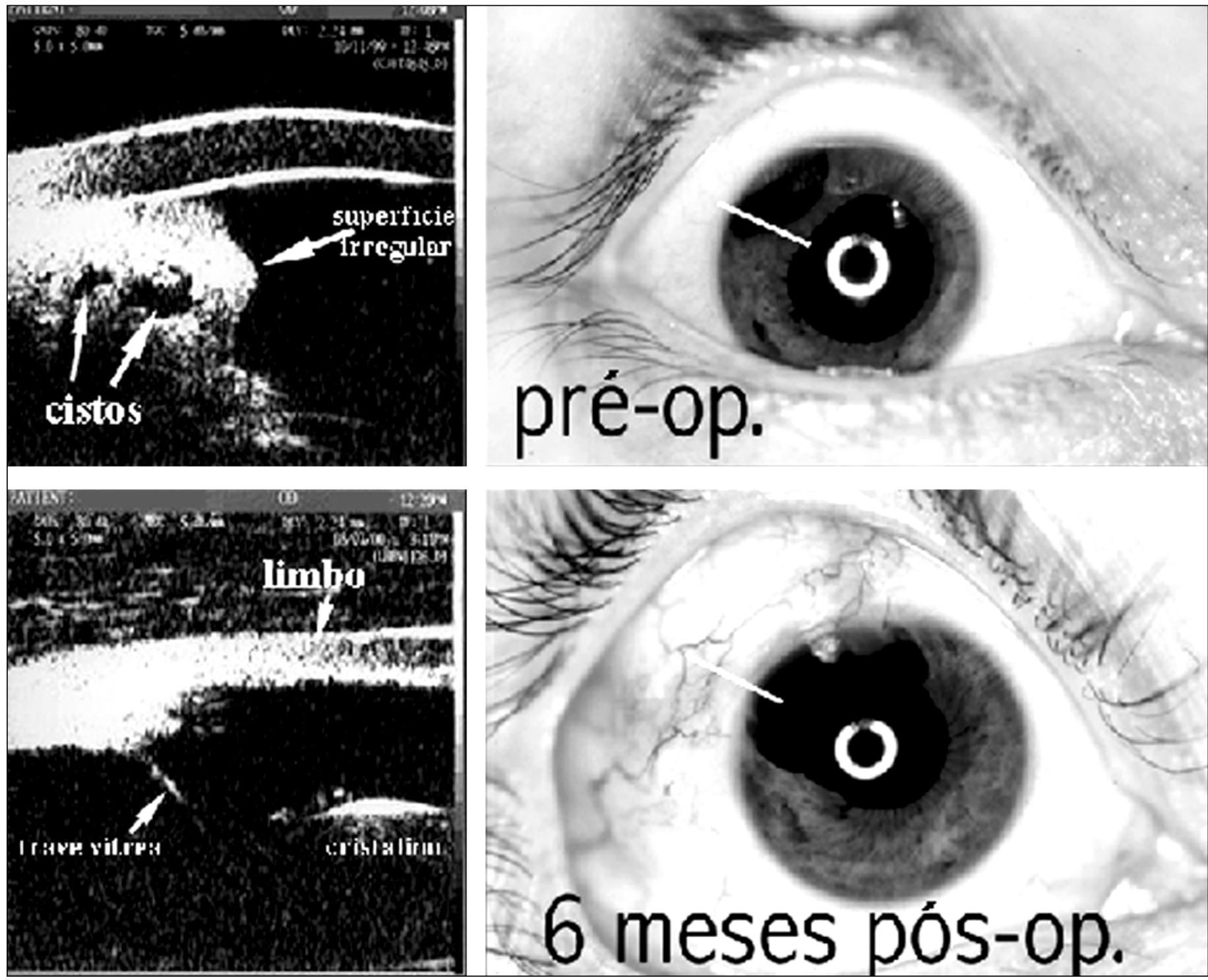

Figura 3 - Biomicroscopia ultra-sônica pré e pós iridociclectomia (caso $n^{0} 1$ ). A ilustração à esquerda superior apresenta massa iridociliar, com 2 cavidades internas de aspecto cístico, preenchendo o ângulo da câmara anterior. Note que a raiz da íris não pode ser distinguida e há comprometimento do corpo ciliar. No UBM, 6 meses depois da ressecção tumoral (iridociclectomia), pode-se observar que a área ressecada e a esclera remanescente apresentam boa espessura (esquerda inferior). A trave vítrea está longe da incisão. As fotografias à direita revelam o aspecto ocular pré e pós-operatório respectivamente.

\section{CONCLUSÃO}

A biomicroscopia ultra-sônica (UBM) pareceu ser um método confiável para diagnosticar, planejar a ressecção cirúrgica e acompanhar o pós-operatório de tumores de íris e corpo ciliar neste pequeno grupo de pacientes. Além disto, as dimensões tumorais reveladas pelo UBM parecem ter relação com a agressividade/tipo histológico do tumor, mas o pequeno número de pacientes nos impede de generalizar estas conclusões.

ABSTRACT

Purpose: To study pre-operatively and post-operatively ul- trasound biomicroscopy (UBM) findings of iris and ciliary body melanomas and correlate tumor extension (revealed by ultrasound biomicroscopy) with histopathology findings. Methods: Prospective study of 5 patients with a clinical diagnosis of iris and ciliary body tumors. These patients were evaluated by ultrasound biomicroscopy before and after treatment with iridectomy or iridociclectomy. All specimens were sent for histopathologic study. Patients were followed-up for a minimum of 6 months when a new ultrasound biomi croscopy examination was performed. Results: In this group of 5 patients, 3 were females and 2, males, age range from 32 to 64 years. All tumors were unilateral. Four patients presented an iridociliary tumor and one presented a lesion limited to the iris. In all cases ultrasound biomicroscopy was very helpful to 
determine surgical planning ensuring free surgical margins. Postoperative ultrasound biomicroscopy revealed no signs of tumor recurrence in all cases; 2 patients presented vitreous in the surgical wound, one with limited vitreous traction but no retinal detachment was detected. Conclusions: Ultrasound biomicroscopy seems to be a reliable ancilliary method to diagnose iris and iridociliary tumors, plan surgical treatment and postoperative follow-up.

Keywords: Ciliary body/ultrasonography; Iris neoplasms/ ultrasonography; Iris neoplasms/surgery; Uveal neoplasms/ ultrasonography; Melanoma; Pigment epithelium of eye/ ultrasonography

\section{REFERÊNCIAS}

1. Katz NR, Finger PT, McCormick SA, Tello C, Ritch R, Sirote M, et al. Ultrasound biomicroscopy in the management of malignant melanoma of the iris. Arch Ophthalmol 1995;113:1462-3.

2. Shields JA, Sanborn GE, Augsburger JJ. The differential diagnosis of malignant melanoma of the iris. A clinical study of 200 patients. Ophthalmology 1983;90:716-20

3. Ayres B, Allemann N, Nakanami C, Adan CBD. Cistos primários do epitélio pigmentar da íris e corpo ciliar: aspectos de biomicroscopia ultra-sônica. Arq Bras Oftalmol 2000;63:337-43.

4. Augsburger JJ, Affel LL, Benarosh DA. Ultrasound biomicroscopy of cystic lesions of iris and ciliary body. Trans Am Ophthalmol Soc 1996;94:259-71; discussion p. 271-4

5. Harbour JW, Augsburger JJ, Eagle RC. Initial management and follow-up of melanocytic iris tumors. Ophthalmology 1995;102:1987-93.

6. Marigo FA, Finger PT, McCormick SA, Iezzi R, Esaki K, Ishikawa H, et al. Iris and ciliary body melanomas: ultrasound biomicroscopy with histopathologic correlation. Arch Ophthalmol 2000;118:1515-21.

7. Augsburger JJ, Lauritzen K, Gamel JW, DeBrakeleer DJ, Lowry JC, Eisenman R. Matched group study of surgical resection versus cobalt-60 plaque radiotherapy for primary choroidal or ciliary body melanoma. Ophthalmic Surg 1990;21:682-8.

8. Allemann N. Biomicroscopia ultra-sônica. Arq Bras Oftalmol 1995;58:283-5.

9. Pavlin CJ, McWhae JA, Mc Gowan HD, Foster FS. Ultrasound biomicroscopy of anterior segment tumors. Ophthalmology 1992;99:1220-8.

10. Corrêa ZM, Augsburger JJ. Ultrasound biomicroscopy of the anterior ocular segment. In: Tasman W, Jaeger EA, editors. Duane's Biomedical Foundation of Ophthalmology. Philadelphia: J.Blippincott; 1997. p. 1-16.

11. Chojniak MMM, Erwenne CM, Allemann N. Biomicroscopia ultra-sônica na avalição de tumores do segmento anterior [abstract]. Arq Bras Oftalmol 1995; 58:256.

12. Corrêa ZMS, Marcon IM. O uso da biópsia aspirativa com agulha fina (BAAF) no diagnóstico de lesão iriana suspeita - relato de caso. Arq Bras Oftalmol 2001;64:347-50.

13. Arentsen JJ, Green WR. Melanoma of the iris: report of 72 cases treated surgically. Ophthalmic Surg 1975;6:23-37.

14. Peyman GA, Raichand M, Schulman J. Diagnosis and therapeutic surgery of the uvea - Part I: Surgical technique. Ophthalmic Surg 1986;17:822-9.

15. McLean IW, Zimmerman LE, Evans RM. Reappraisal of Callender's spindle A type of malignant melanoma of choroid and ciliary body. Am J Ophthalmol 1978;86:557-64.

16. Callender GR. Malignant melanotic tumors of the eye: a study of histologic type in 111 cases. Trans Am Acad Ophthalmol Otolaryngol 1931;36:131-42.

17. Moreira Junior CA, Ramos ARB, Wahab AS, Santos F. Ressecção cirúrgica em bloco de melanoma maligno de coróide. Arq Bras Oftalmol 1996;59:88-91.

\title{
XXIV Congresso do Hospital são Geraldo
}

\section{9 a 11 de outubro de 2003}

\author{
GRANDARRELL MINAS HOTEL \\ BELO HORIZONTE - MC
}

INFORMAÇÕES: Tel/Fax.: (31) 3274-1550

E-mail: comunica@consultcom.com.br

Home page: www.hospitalsaogeraldo.com.br 